Radial and Nonradial Pulsations as Probes of Stellar Physics

ASP Conference Series, Vol. 259, 2002

C. Aerts, T.R. Bedding, \& J. Christensen-Dalsgaard, eds.

\title{
Two-Dimensional Hydrodynamic and Evolution Sequences of Rotating Stars
}

\author{
R. G. Deupree ${ }^{1}$, J. A. Guzik, C. Neuforge-Verheecke \\ Los Alamos National Laboratory, Los Alamos, NM 87545-2345, USA
}

Two-dimensional hydrodynamic simulations were calculated for ZAMS models with $Z=0.02$, and masses of $3,5,8,12$, and $20 \mathrm{M}_{\odot}$. For each mass five models were calculated - one nonrotating and four with progressively higher rotation rates. The rotating models were categorized by the ratio of the polar to the equatorial radius, with values of $0.985,0.92,0.84$, and 0.72 . The simulations were performed with the fully two-dimensional implicit code ROTORC (actually what is known as 2.5 dimensions, with azimuthal symmetry, but with a conservation law for the rotational velocity in the azimuthal direction).

All models showed considerable core overshooting. To first order, the value is about $0.5 \mathrm{H}_{p}$, but with a fairly large variation of about $0.2 \mathrm{H}_{p}$. It is not clear why the variation is so large, but comparison with other turbulence models shows that overshooting is one of the more sensitive features of the model (Deupree, 1998). However, there are some trends that may be significant. For the two highest masses there was apparently no dependence on the rotation rate, but the three smaller masses showed a linear decrease in the extent of overshooting with increasing rotation. There is a hint that the $20 \mathrm{M}_{\odot}$ model has slightly larger overshooting than models of the other masses. With the random variations, it is difficult to assign a level of significance to these trends.

The other significant result is that the rotation rate in the convective core can be fit by a power law in the distance from the rotation axis. Since the power is negative, it must be limited near the polar axis. The power is approximately -0.55 , and the fit is better for the more rapidly rotating models. The results above are presented in Deupree $(2000,2001)$.

We chose two models, both of $20 \mathrm{M}_{\odot}$, for which to calculate stellar evolution sequences. One of these is nonrotating, with which we can compare results not only of the rotating model but also of updated versions of the Paczynski (1970) code, the Iben code $(1963,1965 \mathrm{a}, \mathrm{b})$, and the YREC code (Guenther \& Demarque, 1997, 2000). Since some of these codes do not include overshooting, we ran the ROTORC models without overshooting to make the comparison. The other model was the most rapidly rotating of the $20 \mathrm{M}_{\odot}$ models, which has a ZAMS surface equatorial velocity of $600 \mathrm{~km} \mathrm{~s}^{-1}$. The nonrotating ROTORC evolution sequence agrees with these codes essentially as well as they agree with each other, as there are differences among the physical models of each code.

The evolution tracks of the rotating and nonrotating models are nearly parallel in the HR diagram, although the curves tend toward the same effective

'e-mail: deupree_robert_g@lanl.gov 


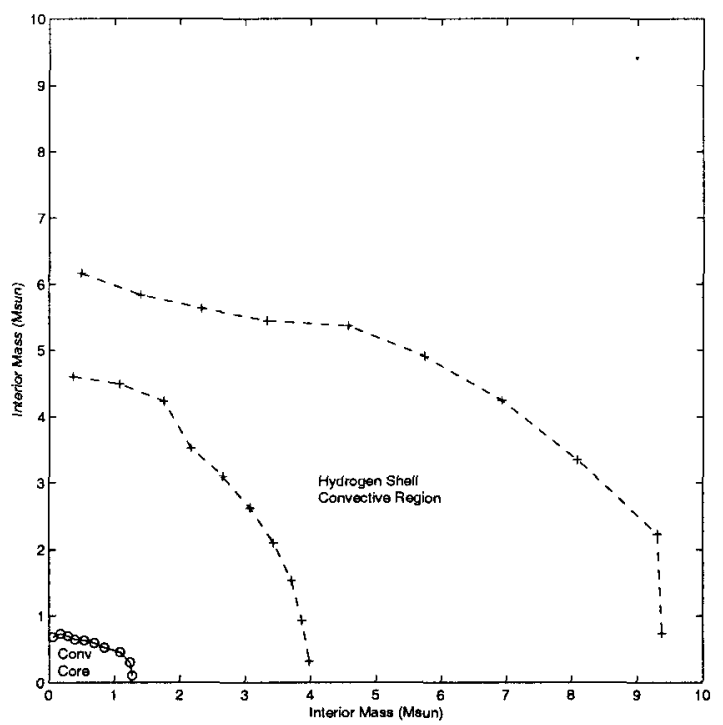

Figure 1. Interior of rotating $20 \mathrm{M}_{\odot}$ model during core He burning. Note the large asymmetry in H-burning shell and He-burning core.

temperature at the first turning point in the evolution. For the rotating model (see Fig. 1), the most interesting features are that 1) the convection zone associated with the hydrogen shell burning is quite aspherical; 2) the convective region associated with core helium burning is highly oblate; and 3) quiescent core helium ignition in the rotating model occurs at an appreciably lower temperature than in the nonrotating model.

These first results indicate that there will be interesting features produced by rotation in these and later stages of evolution, and that future exploration may yield fascinating results.

\section{References}

Deupree, R.G. 1998, ApJ, 499, 340

Deupree, R.G. 2000, ApJ, 543, 395

Deupree, R.G. 2001, ApJ, 552, 268

Guenther, D., Demarque, P. 1997, ApJ, 484, 937

Guenther, D., Demarque, P. 2000, ApJ, 531, 503

Iben, I., Jr. 1963, ApJ, 138, 452

Iben, I., Jr. 1965a, ApJ, 141, 993

Iben, I., Jr. 1965b, ApJ, 142, 1447

Paczynski, B. 1970, Acta Astron., 20, 47 


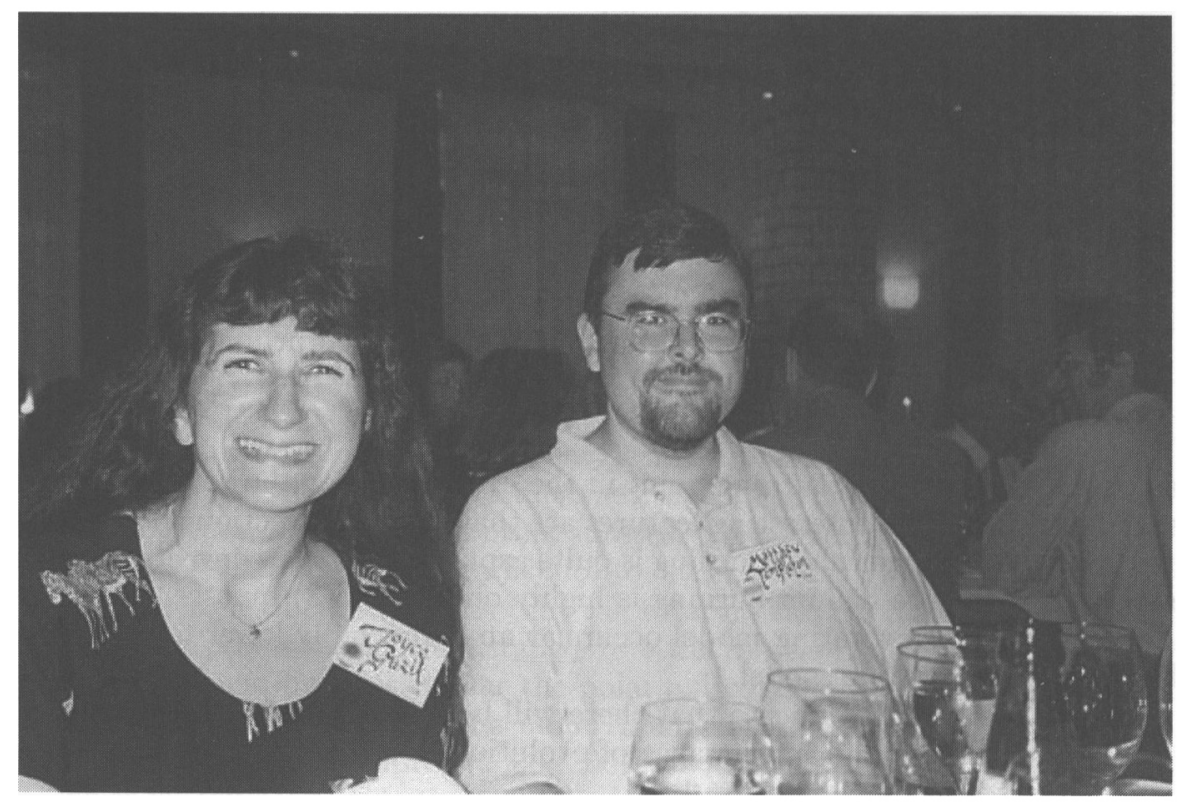

Joyce Guzik and Matthew Templeton are having fun, while the SOC chairman is keeping an eye on his crowd. 